\title{
Annihilation events topology and their generated sound in turbulent premixed flames
}

\author{
D. Brouzet ${ }^{\mathrm{a}, *}$, A. Haghiri ${ }^{\mathrm{a}}$, M. Talei ${ }^{\mathrm{a}}$, M. J. Brear ${ }^{\mathrm{a}}$ \\ ${ }^{a}$ Department of Mechanical Engineering, University of Melbourne, Parkville, VIC 3010, Australia
}

\begin{abstract}
This paper studies the contribution of flame annihilation events to the sound radiated by turbulent, premixed flames. Previously published direct numerical simulation (DNS) datasets of stoichiometric and lean $(\phi=0.7)$ flames (Haghiri et al. 2018) are first examined using an efficient formulation of the method of Griffiths et al. (2015) to identify the annihilation events. Four classes of annihilation event are observed. Three of these - pocket burn-out, tunnel closure and tunnel formation - were defined by Griffiths et al. A 'multi-feature' event is also defined in this paper as any combination of the other three annihilation events occurring close enough such that their radiated sound can be considered as originating from a single event.

Further post-processing of these stoichiometric and lean datasets shows that the fluctuations in heat release rate associated with these 4 observed types of annihilation events are responsible for the broadband sound radiated by both flames. This, in turn, suggests that flame annihilation is the physical mechanism by which air-fuel ratio affects the radiated sound amplitude at high frequencies. This result is supported by previous works which have shown that the sound radiated from individual annihilation events scales with the laminar flame speed and the temperature ratio.
\end{abstract}

Keywords: Combustion noise, Annihilation event, Turbulent premixed flame

\section{Introduction}

Commercial and regulatory requirements continue to demand increased efficiency and reduced emissions from gas turbines. Lean premixed combustion is a means of achieving these goals. However, combustors operating under lean premixed conditions are susceptible to socalled 'thermo-acoustic instabilities', which is an unstable coupling between the flame and the combustor acoustics [1-3]. One mechanism that leads to this coupling involves sound generation by the flame only, referred to as 'direct combustion noise' [4-9]. Therefore, achieving a better understanding of the sound production by premixed flames is important for mitigating these instabilities.

Early studies on combustion noise appeared in the 1960's and involved experimental and theoretical investigations. Various authors [10-13] suggested that the main source of noise in open

${ }^{*}$ Corresponding author

Email addresses: brouzetd@student .unimelb.edu.au (D. Brouzet), haghiri.a@unimelb.edu.au (A. Haghiri), mohsen.talei@unimelb.edu.au (M. Talei), mjbrear@unimelb.edu.au (M. J. Brear)

Preprint submitted to Combustion and Flame 
premixed, turbulent flames were monopolar sources inside the reaction zone. The experimental study of Smith \& Kilham [11] revealed the broadband nature of combustion noise, with the frequency of peak amplitude and the total acoustic power depending on the inlet flow velocity, the burner diameter and the laminar flame speed. These dependencies were confirmed in several subsequent studies (e.g. [14-18]), although these works proposed different scalings for the radiated sound spectra.

The importance of heat release rate fluctuations to the sound generation process has also been highlighted by many authors (e.g. $[8,9,19-21])$. One mechanism that may lead to rapid fluctuations of the heat release rate is flame annihilation $[22,23]$. When two flame surfaces collide, the unburned gas trapped between them is rapidly consumed, resulting in quick variations of the local heat release rate. Thomas \& Williams [22] were the first to recognize that an inwardly radially propagating flame, leading to flame annihilation, produces pressure fluctuations. They also emphasized the importance of the flame displacement speed in the sound generation process. Several experimental studies subsequently investigated the significance of annihilation events in the generation of sound by forced laminar premixed flames. Kidin et al. [24, 25] analyzed sound produced by tunnels and bubbles of reactants being consumed. Both events were found to be associated with acoustic pressure peaks in the far field and Kidin's simple theoretical model relating annihilation to the radiated sound was reported to be in reasonable agreement with experimental observations.

More recently, Schuller et al. [5] showed that strong variations of the flame surface area due to annihilation events could lead to self sustained oscillations of the flame front. If the pressure fluctuation produced by the annihilation event was in phase with the velocity fluctuations at the burner's inlet, the flame would be unstable, showing therefore their potential involvement in flame instabilities. Using forced laminar premixed flames to study sound produced by flame/plate, flame/flame and flame/vortex interactions, Candel et al. [26] then found that those three interactions were strong sources of noise, and that mutual flame annihilation could be a major source of noise in turbulent combustors.

Following these experimental investigations, several numerical studies on annihilation events have since been published. Talei et al. $[23,27]$ analyzed the sound produced by planar, axisymmetric and spherically symmetric flame annihilations in a one-dimensional (1-D), laminar, premixed flame. They also developed a theoretical framework for calculating the sound produced. In subsequent studies, flame annihilation was also observed as a significant source of sound in two-dimensional (2-D) forced laminar premixed flames [28, 29]. More recently, Haghiri et al. [30] performed direct numerical simulation (DNS) of sound generation by turbulent premixed flames with equivalence ratios of $\phi=1.0$ and $\phi=0.7$. They confirmed that annihilation events were a significant source of noise and developed a simple model to estimate the contribution of annihilation events to the far-field sound.

However, the simple theory of Haghiri et al. [30] did not distinguish between different types of annihilation events and their relative contributions to the far-field noise. This study therefore reconsiders the dataset of Haghiri et al. [30] by using the method proposed by Griffiths et al. [31] for finding different forms of flame interaction events. We then relate these events to the overall far-field sound produced by the flame.

\section{DNS dataset}

The compressible DNS used in this study was performed using a version of S3D [32] featuring a simple chemistry scheme, referred to as S3D-SC [33-35]. This combustion solver uses an 


\begin{tabular}{lccc}
\hline Quantity & Units & $\phi=1.0$ & $\phi=0.7$ \\
\hline$D$ & $\mathrm{~mm}$ & 3.94 & 4.63 \\
$c_{\text {ref }}$ & $\mathrm{m} \cdot \mathrm{s}^{-1}$ & 562 & 560.8 \\
$\rho_{\text {ref }}$ & $\mathrm{kg} \cdot \mathrm{m}^{-3}$ & 0.421 & 0.426 \\
$T_{\text {ref }}$ & $\mathrm{K}$ & 800 & 800 \\
\hline \multicolumn{4}{r}{} \\
\end{tabular}

Table 1: Inlet values used for non-dimensionalisation.

$8^{\text {th }}$ order central differencing scheme for spatial derivatives, combined with a 6 -stage, $4^{\text {th }}$ order explicit Runge-Kutta time integrator.

The DNS featured a subsonic round jet of unburned premixed mixture issuing into an open environment of combustion products at the adiabatic flame temperature. Methane/air combustion was considered at two different equivalence ratios $(\phi=1.0$ and $\phi=0.7)$, with the reactants preheated to $800 \mathrm{~K}$ at $1 \mathrm{~atm}$. The jet Reynolds number was equal to 5300 for both cases and a synthetic turbulence field with a turbulence intensity of $3.7 \%$ at the jet centerline was fed into the mean velocity field using Taylor's hypothesis. A single step, irreversible chemistry model based on Arrhenius' law was used to reduce the computational cost with the fuel mass fraction $Y_{f}$ defined as progress variable. The single-step model used matched several important parameters including the laminar flame speed, the flame thickness and the temperature ratio. We refer to the original paper from Haghiri et al. [30] for more details about the single-step chemistry model.

Dimensional quantities, represented with the superscript ' $*$ ', are non-dimensionalised using inlet values (see table 1), which are denoted by the ref subscript:

$$
\begin{gathered}
\mathbf{x}=\frac{\mathbf{x}^{*}}{D}, \quad t=\frac{t^{*} c_{r e f}}{D}, \quad \mathbf{u}=\frac{\mathbf{u}^{*}}{c_{r e f}}, \quad \rho=\frac{\rho^{*}}{\rho_{r e f}} \\
T=\frac{T^{*}}{(\gamma-1) T_{r e f}}, \quad p=\frac{p^{*}}{\rho_{r e f} c_{r e f}^{2}}
\end{gathered}
$$

where $D$ is the jet diameter, $t$ is the time, $c$ is the sound speed, $\mathbf{u}$ is the velocity vector, $\rho$ is the density, $T$ is the temperature, $\gamma$ is the heat capacity ratio (considered to be 1.4) and $p$ is the pressure. The spatial coordinate vector is represented as $\mathbf{x}=[x, y, z]$, with $x, y$ and $z$ denoting the streamwise, tranverse and spanwise coordinates, respectively. In the following, subscripts in and $b$ correspond to dimensionless values at the inlet and in the burnt gas, respectively, and the overline - refers to the temporal ensemble average.

The simulation was performed on a three-dimensional (3-D) structured cartesian mesh, with a grid size in the jet region that was 14 times smaller than the laminar thermal flame thickness $\delta_{t h}$. All non-reflecting outflow boundaries were carefully treated to avoid spurious noise reflections. The boundary conditions were based on the so-called 'Navier-Stokes Characteristic Boundary Conditions' (NSCBC) [36, 37]. Moreover, a sponge region [38] was added from $x=20$ to $x=25$ to gradually damp the velocity fluctuations at the outflow boundary.

The data were collected for about 1.3 flow-through times with a sufficient time resolution for capturing annihilation events. Table 2 summarizes the DNS parameters and a representation of the $\phi=1.0$ flame with the iso-surface of maximum reaction rate can be seen in Fig. 1 . More details on the flow configuration and numerical setup can be found in the original paper of Haghiri et al. [30]. 


\begin{tabular}{lcc}
\hline Parameters & $\phi=1.0$ & $\phi=0.7$ \\
\hline Domain size $\left(L_{x} \times L_{y} \times L_{z}\right)$ & \multicolumn{2}{c}{$20 D \times 16 D \times 16 D$} \\
Grid resolution $\left(N_{x} \times N_{y} \times N_{z}\right)$ & $2412 \times 1040 \times 1040$ \\
Mean inlet Mach number $\left(M=\bar{u}_{i n} / c_{i n}\right)$ & 0.35 & 0.26 \\
Heat release parameter $(\alpha)$ & 0.675 & 0.637 \\
Jet Reynolds number & & \\
$\left(R e=\bar{u}_{\text {in }} D / v\right)$ & 5300 & 5300 \\
Inlet turbulent intensity $\left(u^{\prime} / \bar{u}_{\text {in }}\right)$ & $3.7 \%$ & $3.7 \%$ \\
Kolmogorov scale $(\eta)$ & 0.014 & 0.014 \\
Turbulent Reynolds number $\left(R e_{T}=u^{\prime} l_{t} / v\right)$ & 58.8 & 58.8 \\
Thermal flame thickness $\left(\delta_{t h}\right)$ & 0.07 & 0.07 \\
Laminar flame speed $\left(S_{L}\right)$ & $4.22 \cdot 10^{-3}$ & $3.25 \cdot 10^{-3}$ \\
Flow through time & 57 time units & 77 time units \\
Zeldovich number $(\beta)$ & 7.9 & 5.5 \\
Damkohler number $(D a)$ & 8.6 & 3.24 \\
Prandtl number $(P r)$ & 0.72 & 0.72 \\
Lewis number $(L e)$ & 1 & 1 \\
\hline
\end{tabular}

Table 2: Parameters of the DNS dataset from Haghiri et al. [30]. All quantities are dimensionless. The streamwise flow velocity, the sound speed, the kinematic viscosity and the integral length scale are represented by $u, c, v$ and $l_{t}$, respectively.

\section{Methodology}

\subsection{Acoustic theory}

Lighthill's acoustic analogy is commonly used to study sound generation by turbulent flows [39]. This equation is a rearrangement of the continuity and momentum equations into a wavelike equation as follows:

$$
\frac{1}{c_{b}^{2}} \frac{\partial^{2} p}{\partial t^{2}}-\nabla^{2} p=\frac{\partial}{\partial x_{i} \partial x_{j}}\left(\rho u_{i} u_{j}-\tau_{i j}\right)-\frac{\partial^{2} \rho_{e}}{\partial t^{2}}
$$

where $\tau$ is the viscous stress tensor and $\rho_{e}$ is the excess density as defined in [21] :

$$
\rho_{e}=\rho-\bar{\rho}_{b}-\left(p-\bar{p}_{b}\right) / \bar{c}_{b}^{2}
$$

The right hand side of Eq. (2) can be reformulated such that the rate of change of heat release rate $\partial \dot{Q} / \partial t$ appears as one of the source terms. In the context of low Mach number, open, turbulent premixed flames, direct combustion noise induced by $\partial \dot{Q} / \partial t$ has been demonstrated to be the dominant source of sound (e.g. $[12,19,21])$. This is also verified in section 4.2 of this paper for the considered dataset. Assuming that this term is dominant we obtain,

$$
\frac{1}{c_{b}^{2}} \frac{\partial^{2} p}{\partial t^{2}}-\nabla^{2} p \approx \frac{\gamma-1}{c_{b}^{2}} \frac{\partial \dot{Q}}{\partial t}
$$




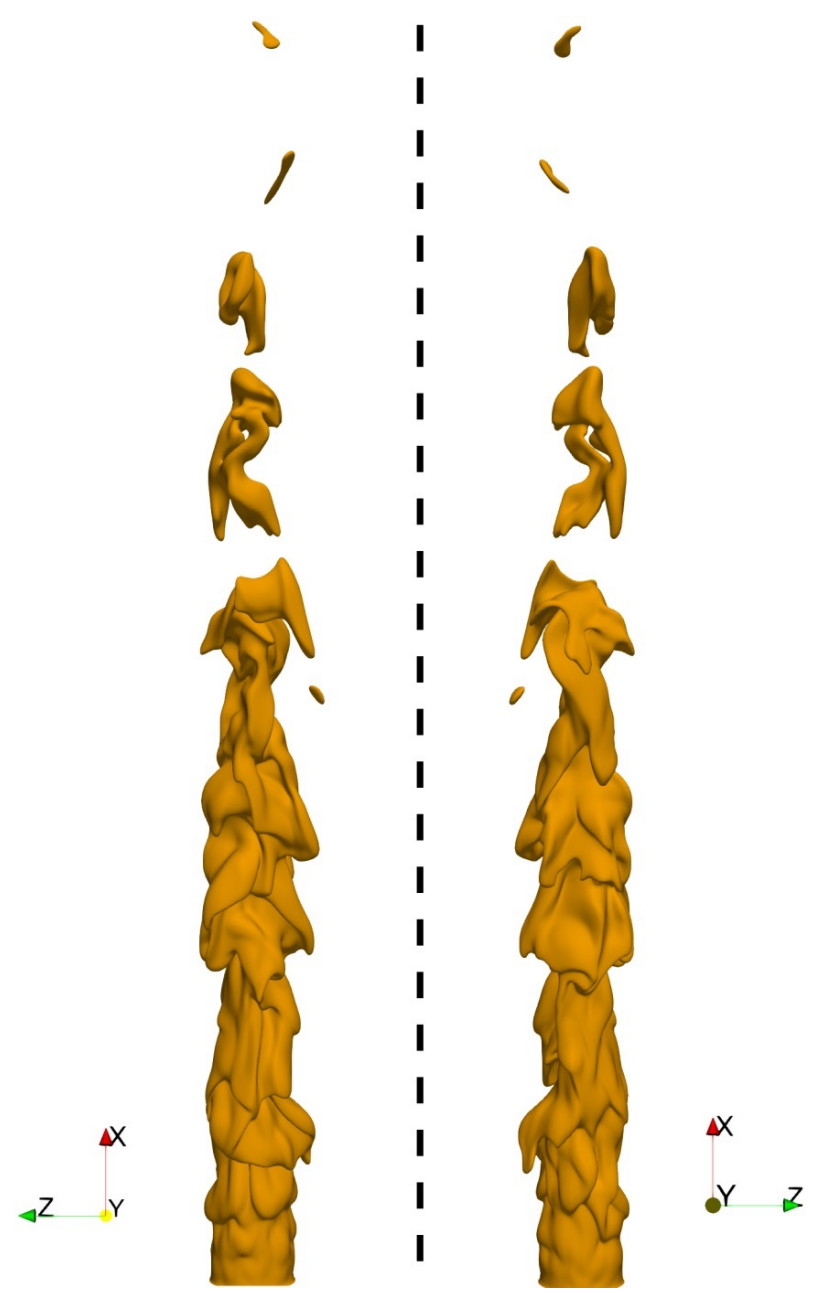

Figure 1: Front and rear views of the $\phi=1.0$ turbulent premixed flame represented by the iso-surface of maximum reaction rate.

The contribution of $\partial \dot{Q} / \partial t$ to the far-field pressure fluctuations $p^{\prime}$ can be determined numerically using a free space Green's function,

$$
p^{\prime}(\mathbf{x}, t) \approx \frac{\gamma-1}{c_{b}^{2}} \sum_{i=1}^{N_{\text {cells }}} \frac{\partial \dot{Q} / \partial t\left(\mathbf{x}_{i}, t-\frac{\left|\mathbf{x}-\mathbf{x}_{i}\right|}{c_{b}}\right) \cdot V_{i}}{4 \pi\left|\mathbf{x}-\mathbf{x}_{i}\right|}
$$

where $N_{\text {cells }}$ is the total number of computational cells, and $\mathbf{x}_{i}$ and $V_{i}$ are the centre and volume of the $i^{t h}$ cell, respectively. As $\partial \dot{Q} / \partial t$ is not explicitly computed in the DNS, an analytical expression is derived to compute it. For unity Lewis number, Poinsot \& Veynante [7] explain that

$$
\theta+Y_{f}=1
$$

where $Y_{f}$ is the fuel mass fraction and $\theta$ is the reduced temperature defined as $\theta=\left(T-T_{i n}\right) /\left(T_{b}-\right.$ 
$\left.T_{i n}\right)$. We can therefore express the heat release rate $\dot{Q}$ as

$$
\dot{Q}=-\Delta h_{f}^{o} \dot{\omega}_{f}=\Delta h_{f}^{o}\left[\rho D a Y_{f} \exp \left(\frac{-\beta Y_{f}}{1-\alpha Y_{f}}\right)\right],
$$

where $\dot{\omega}_{f}$ is the fuel reaction rate, $\Delta h_{f}^{o}$ is the fuel's enthalpy of formation, $D a$ is the Damkohler number (which corresponds to the dimensionless pre-exponential factor from the Arrhenius law), $\alpha$ is the heat release rate parameter and $\beta$ is the Zeldovich number. The time derivative of $\dot{Q}$ can be expressed using the chain rule,

$$
\frac{\partial \dot{Q}}{\partial t}=\frac{\partial \dot{Q}}{\partial \rho} \frac{\partial \rho}{\partial t}+\frac{\partial \dot{Q}}{\partial Y_{f}} \frac{\partial Y_{f}}{\partial t} .
$$

The two derivatives $\partial \dot{Q} / \partial \rho$ and $\partial \dot{Q} / \partial Y_{f}$ can be easily derived from Eq. (7). The two terms $\partial \rho / \partial t$ and $\partial Y_{f} / \partial t$ are estimated using the continuity and the species equations, respectively:

$$
\begin{gathered}
\frac{\partial \rho}{\partial t}=-\nabla \cdot(\rho \mathbf{u}), \\
\frac{\partial Y_{f}}{\partial t}=-\mathbf{u} \cdot \nabla Y_{f}+\frac{1}{\rho} \nabla \cdot\left(\rho D_{f} \nabla Y_{f}\right)+\frac{\dot{\omega}_{f}}{\rho},
\end{gathered}
$$

where $D_{f}$ the mass diffusion coefficient of the fuel. Using Eq. (7) to (10), $\partial \dot{Q} / \partial t$ is then computed without any knowledge of the previous or next timesteps,

$$
\begin{aligned}
\frac{\partial \dot{Q}}{\partial t}=\underbrace{-\frac{\dot{Q}}{\rho} \nabla \cdot(\rho \mathbf{u})}_{\text {I }}+f\left(\rho, Y_{f}\right) \\
\quad \times[\underbrace{\frac{\dot{\omega}_{f}}{\rho}}_{\text {II }}-\underbrace{\mathbf{u} \cdot \nabla Y_{f}}_{\text {III }}+\underbrace{\frac{1}{\rho} \nabla \cdot\left(\rho D_{f} \nabla Y_{f}\right)}_{\text {IV }}],
\end{aligned}
$$

where

$$
f\left(\rho, Y_{f}\right)=\Delta h_{f}^{o} \rho D a \exp \left(\frac{-\beta Y_{f}}{1-\alpha Y_{f}}\right)\left(1-\frac{\beta Y_{f}}{\left(1-\alpha Y_{f}\right)^{2}}\right) .
$$

Term I in Eq. (11) expresses the effect of compressibility on the rate of change of the heat release rate. Terms from II to IV represent respectively the contribution of the reaction rate, convection and diffusion. The term $\partial \dot{Q} / \partial t$ can be also expressed as a function of the displacement speed $S_{d}[40]$ :

$$
\frac{\partial \dot{Q}}{\partial t}=-\frac{\dot{Q}}{\rho} \nabla \cdot(\rho \mathbf{u})-f\left(\rho, Y_{f}\right)\left(\mathbf{u}+S_{d} \mathbf{n}\right) \cdot \nabla Y_{f},
$$

where $\mathbf{n}$ is the flame's normal vector $\nabla Y_{f} /\left|\nabla Y_{f}\right|$ and

$$
S_{d}=-\frac{\dot{\omega}_{f}}{\rho\left|\nabla Y_{f}\right|}-\frac{\nabla \cdot\left(\rho D_{f} \nabla Y_{f}\right)}{\rho\left|\nabla Y_{f}\right|} .
$$

Equation 13 shows the potential of an increased flame displacement speed to quickly change the heat release rate and therefore, to be a strong source of sound, as shown by several authors (e.g $[23,26,30])$. 


\subsection{Identifying flame annihilation}

The method proposed by Griffiths et al. [31] is used to identify the location of annihilation events. Considering that flame annihilation occurs when two flame surfaces collide, a critical point in the progress variable field will be formed. The critical point, defined as the location where the spatial gradient of the progress variable is zero, can then be used to represent an annihilation event.

Figure 2 shows that to find critical points, we first performed a linear interpolation of the gradient vector for each computational cell using the values at its 8 vertices. If this indicated that a given cell contained a point with the gradient of the progress variable equalling zero, a Newton's optimization was performed to calculate the location of the critical points, using the centre of the cell as the first guess. Iteration using Newton's step formula was performed until convergence is reached (see Appendix A for more details about the interpolation method used in Newton's algorithm). Once the critical point had been found, it was used only if the conditions discussed later in section 3.3 were satisfied.

The critical points were then tracked in time in order to analyze the statistics related to the flame annihilation events. The convection velocity at the critical points from the previous timestep was used to estimate their current position. If any current critical points matched one of those estimations, it was assumed that this point was the same annihilation event that had been convected by the flow.

It should be noted that several annihilation events might occur simultaneously at different spatial locations. The number of annihilation events occurring at a given time varied from 1 to 18 , therefore resulting in a total number of events shown in table 3.

\subsection{Conditioning flame annihilation events}

Even though all annihilation events feature a critical point on a flame iso-surface, not all annihilation events are a significant source of noise. The following conditions were therefore imposed on the annihilation event algorithm:

1. The critical point was in a given $Y_{f}$ range, and

2. $\partial \dot{Q} / \partial t$ at the critical point was following the behaviour of a normally propagating annihilation event.

The first condition removed annihilation events in which their rate of change of heat release rate was close to zero. Using 1-D laminar flame simulations of annihilation events under the same conditions as the DNS cases, we determined the range of $Y_{f}$ corresponding to small values of the $\partial \dot{Q} / \partial t$. A threshold of $5 \%$ of the maximum spatially integrated $\partial \dot{Q} / \partial t$ was used. This corresponds to $Y_{f}=[0.002,0.35]$ and $Y_{f}=[0.01,0.45]$ for the $\phi=1$ and $\phi=0.7$ cases, respectively.

The second condition also removed critical points that were not associated with significant generation of sound. An annihilation event at a constant propagation speed features a positive $\partial \dot{Q} / \partial t$ for $Y_{f}>Y_{f-p e a k}$, where $Y_{f-p e a k}$ refers to the point of maximum reaction rate. Outside this range, a negative $\partial \dot{Q} / \partial t$ is observed. To demonstrate this, the red line in Fig. 3 denotes a planar annihilation event at a constant propagation speed of $10 S_{L}$ as an example. We track all the critical points in time and those which belong to the same event should all satisfy the following conditions:

$$
\begin{aligned}
& \partial \dot{Q} / \partial t>0, \text { if } Y_{f}>Y_{f-\text { peak }} \text { and } \\
& \partial \dot{Q} / \partial t<0, \text { if } Y_{f}<Y_{f-\text { peak }}
\end{aligned}
$$




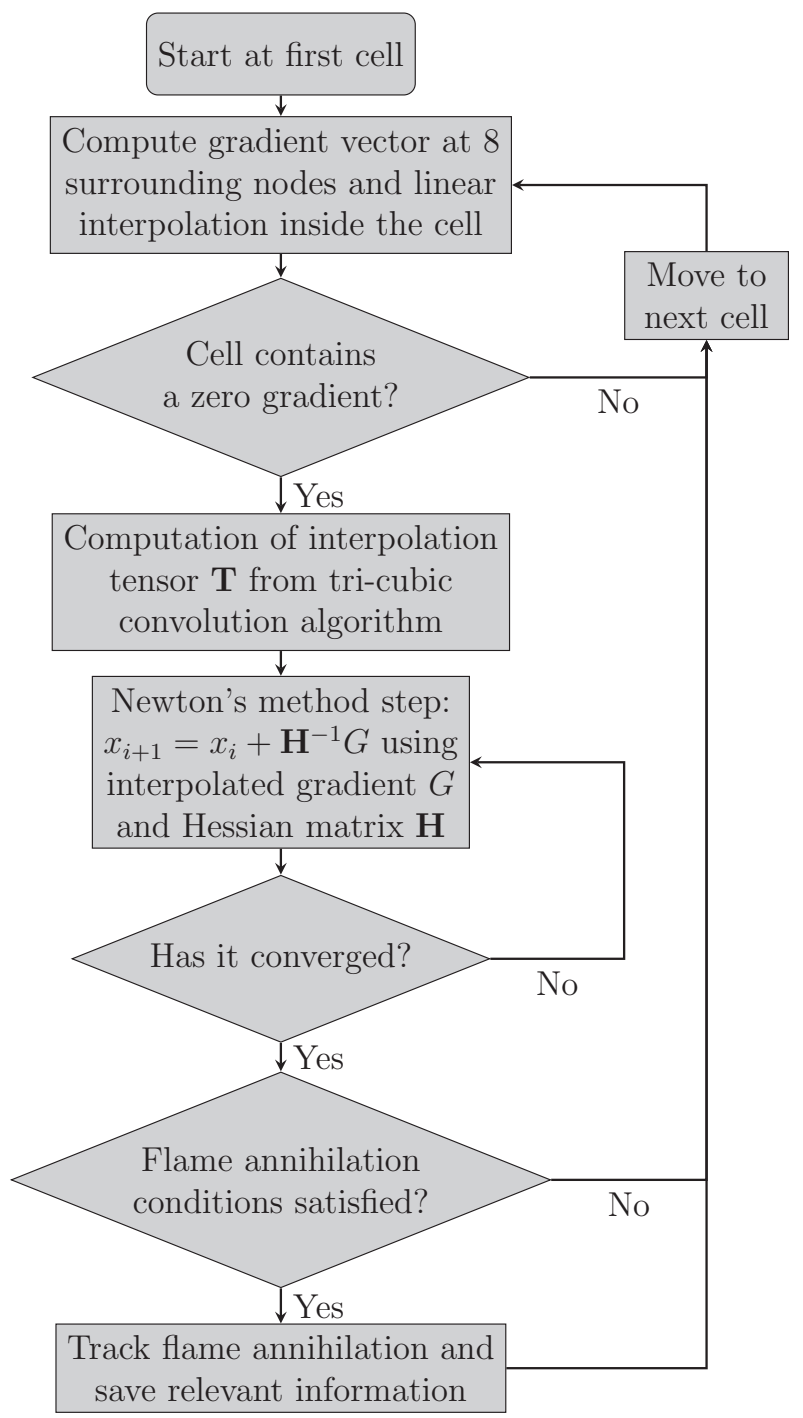

Figure 2: Flowchart for identifying flame annihilation events.

Figure 3 shows $\partial \dot{Q} / \partial t$ at all critical points in the $Y_{f}$ range of interest for the $\phi=1.0$ flame. As can be seen, this conditioning identifies the critical points with high values of $\partial \dot{Q} / \partial t$.

\subsection{Topologies of annihilation events}

Figure 4 shows the four different 3-D flame interaction topologies defined by Griffiths et al. [31], namely 'reactant pocket', 'tunnel formation', 'tunnel closure' and 'pocket burn-out'. The reactant pocket features a region of burnt gas with the flame propagating outwards whereas a pocket burn-out is a pocket of unburnt gases with the flame propagating inwards. Tunnel formation occurs when a hole appears in the progress variable iso-surface due to the interaction of 


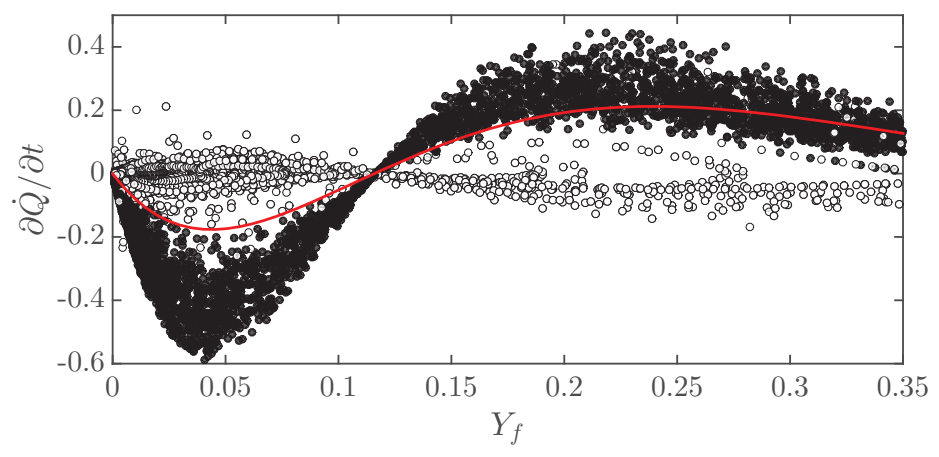

Figure 3: $\partial \dot{Q} / \partial t$ at all critical points in the $Y_{f}$ range of interest for $\phi=1.0$. Flame annihilation events satisfying the second $\partial \dot{Q} / \partial t$ condition are shown with full black circles whereas the remaining events are represented by hollow white circles. The red line represents the $\partial \dot{Q} / \partial t$ that results from a planar annihilation at a constant propagation speed of $10 S_{L}$.

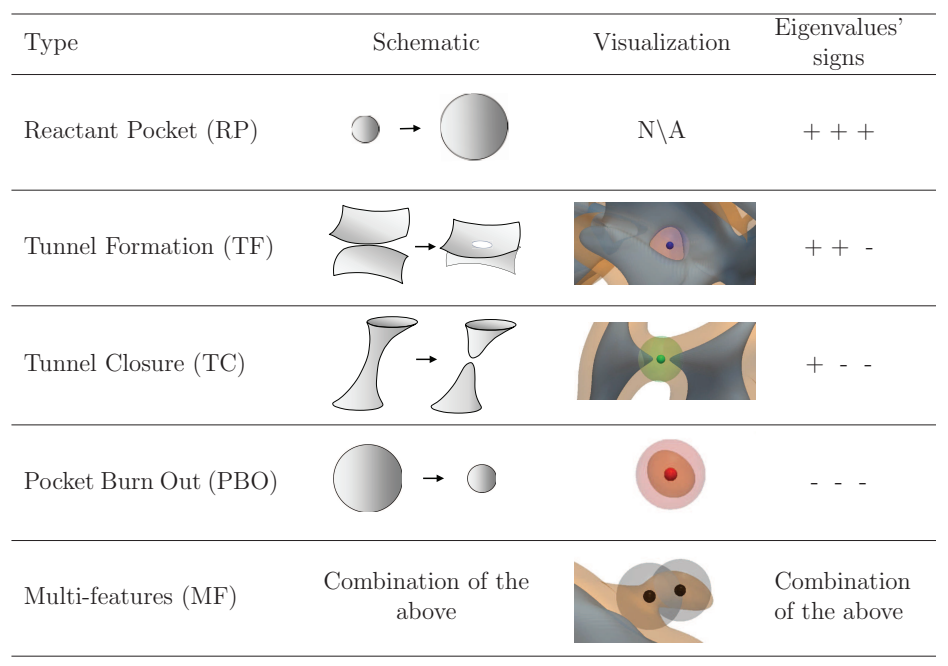

Figure 4: Different interaction topologies.

two flame surfaces. Finally, a tunnel closure appears when a pocket of unburnt gas pinches off from the flame, resulting in two distinct unburnt regions. These different topologies are identified through analysis of the eigenvalues of the Hessian matrix at the critical point, as shown in Fig. 4.

Another type of interaction topology is defined in this paper when several of the canonical topologies described earlier occurred less than two flame thicknesses apart, so that their radiated sound can be considered as originating from a single event. This category is termed 'multifeature' interactions.

Figure 5 shows a snapshot of the stoichiometric flame as well as all four types of interaction topologies found in the dataset, i.e. tunnel formation, tunnel closure, pocket burn-out and multifeature interactions. No reactant pockets were observed since in flames with a moderate level of turbulence, reactant pockets only appear during auto-ignition events, which do not occur in a stable premixed flame. The four topologies found in the dataset mainly destruct flame surface, 
a)

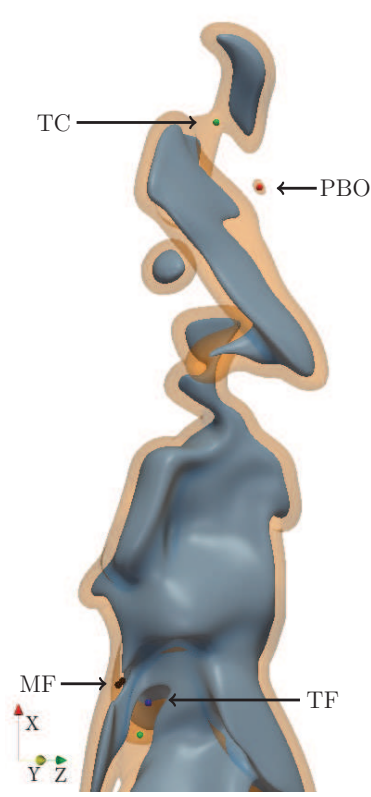

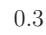

0.6

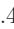

0.8

1.2

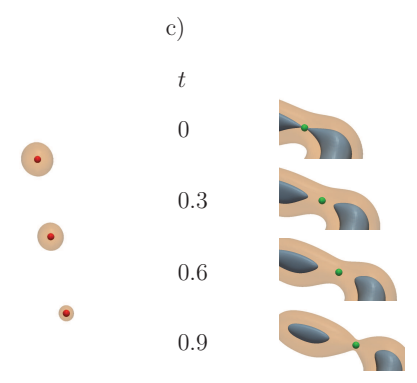

e)

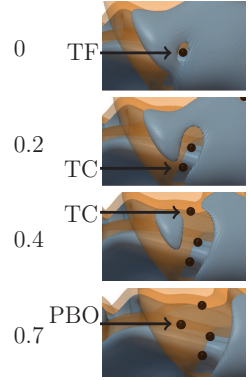

Figure 5: a) A snapshot of the flame and examples of b) pocket burn-out (PBO), c) tunnel closure (TC), d) tunnel formation (TF) and e) multi-feature annihilation events (MF) for $\phi=1.0$. The two iso-surfaces represent the progress variable range used in the searching algorithm.

as it will be shown in the results section, and are therefore labelled as 'annihilation events' in the following. The multi-feature event shown in Fig. 5e was initiated with a tunnel formation, which led to two tunnel closures. These closure events then resulted in formation of a separate pocket of unburnt gas, leading to a pocket burn-out event.

\subsection{Defining annihilation regions}

To determine the contribution of annihilation events to the total generated sound, one needs to define a region around a given critical point that accounts for the associated $\partial \dot{Q} / \partial t$. For an isolated pocket burn out, a sphere with its centre located at the critical point is an obvious approach. However, it was not straightforward to define such regions for other topologies. Acknowledging this complexity, we therefore define the region of significant heat release around each critical point as a sphere with $3 \delta_{t h}$ diameter after significant sensitivity analysis and noting that the results presented later in this paper suggest that this is a reasonable choice. The transparent spheres in Fig. 4 represent those regions, which are called 'annihilation regions' in this work.

\section{Results and discussion}

\subsection{Dynamics of annihilation events}

The location of flame annihilation events with different topologies for the $\phi=1$ and $\phi=$ 0.7 cases are shown in Fig. 6. This figure shows that all four types of annihilation events are 


\begin{tabular}{lcc}
\hline Type & $\phi=1$ & $\phi=0.7$ \\
\hline Pocket burn out & $50(28.5 \%)$ & $67(29 \%)$ \\
Tunnel closure & $85(48.6 \%)$ & $115(49.7 \%)$ \\
Tunnel formation & $40(22.9 \%)$ & $49(21.3 \%)$ \\
Multi-feature & 84 & 157 \\
\hline
\end{tabular}

Table 3: Number of canonical and multi-feature annihilation events per jet flow through time. The percentages take into account the canonical events only.

\begin{tabular}{lcc}
\hline Type & $\phi=1$ & $\phi=0.7$ \\
\hline Multi-feature & 84 & 157 \\
* Pocket burn out & $37(20.6 \%)$ & $70(21.5 \%)$ \\
* Tunnel closure & $90(50.5 \%)$ & $166(50.8 \%)$ \\
* Tunnel formation & $51(28.9 \%)$ & $90(27.7 \%)$ \\
\hline
\end{tabular}

Table 4: Composition of multi-feature annihilation events.

distributed across both the stoichiometric and lean flames. Comparison of the $\phi=1$ and $\phi=0.7$ cases shows a similar behaviour between the two flames in terms of distribution of different types of events. Different types of events start to occur from $x / L_{\text {flame }}=0.2$, where $L_{\text {flame }}$ is the average flame length. However, the average streamwise location of the tunnel formation events is closer to the nozzle exit than that of the tunnel closure events. The average location of the pocket burnout events is observed to be further downstream. This is expected because a tunnel formation commonly leads to one or more tunnel closures, followed by one or more pocket burn-outs, as seen in Fig. 5e. Closer inspection of the results showed that tunnel formation $\rightarrow$ tunnel closure $\rightarrow$ pocket burn-out and tunnel closure $\rightarrow$ pocket burn-out were by far the most common event sequences. It is also interesting to note that the multi-feature events are distributed similarly for each type.

The normalized flame displacement speed $S_{d} / S_{L}$ is now computed for each annihilation event. As the critical points result in a displacement speed singularity, the mean $\overline{S_{d}}$ at the progress variable iso-surface of maximum reaction rate in the annihilation region is computed. The resulting histogram of the mean $\overline{S_{d}} / S_{L}$ is shown in Fig. 7, showing that the displacement speed is commonly an order of magnitude larger than the laminar flame speed. As shown by Eq. (5) and Eq. (13) and discussed later, large flame displacement speeds will result in rapid fluctuations of the heat release rate and, therefore significant pressure fluctuations.

Table 3 shows the number of annihilation events, classified by their topology, per jet flow through time for the $\phi=1.0$ and $\phi=0.7$ cases. The percentages shown in brackets represent the relative frequency of each canonical event type. Tunnel closures and multi-feature events occur more frequently while pocket burn-outs and tunnel formations are less frequent. Closer inspection of multi-feature events indicate that they most commonly include either a combination of a tunnel formation and a tunnel closure or a tunnel closure and a pocket burn-out (Table 4). All annihilation events are also more common in the $\phi=0.7$ case. This is perhaps surprising since the flames have the same Reynolds number and turbulent Reynolds number (table 2), and is likely due to the lean flame having a more wrinkled flame surface due to weaker relaminarisation associated with its lower flame temperature. 

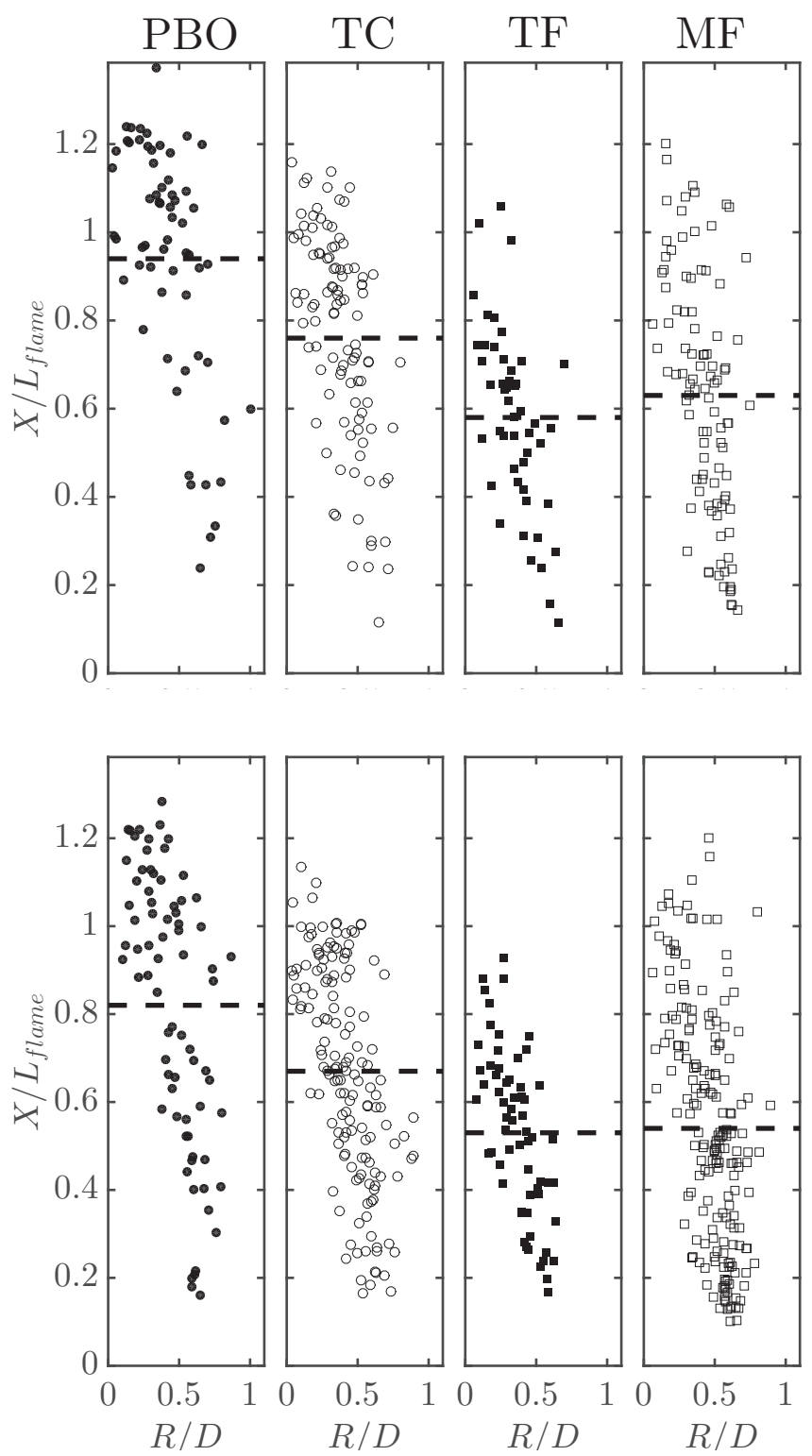

Figure 6: Location of flame annihilation events for pocket burn-outs (full circle), tunnel closures (hollow circle), tunnel formations (full square) and multi-feature events (hollow square) in the $\phi=1.0$ (top) and $\phi=0.7$ (bottom) flames. The dashed lines represent the average streamwise location of the events.

The ensemble averaged pressure fluctuations for the three canonical types of annihilation event at their critical points are plotted in Fig. 8. The reference pressure was chosen when the integral of $\partial \dot{Q} / \partial t$ in the annihilation region was equal to zero. The pocket burn out (black circles) and tunnel closure (white circles) events exhibit similar pressure fluctuations. The pressure fluc- 

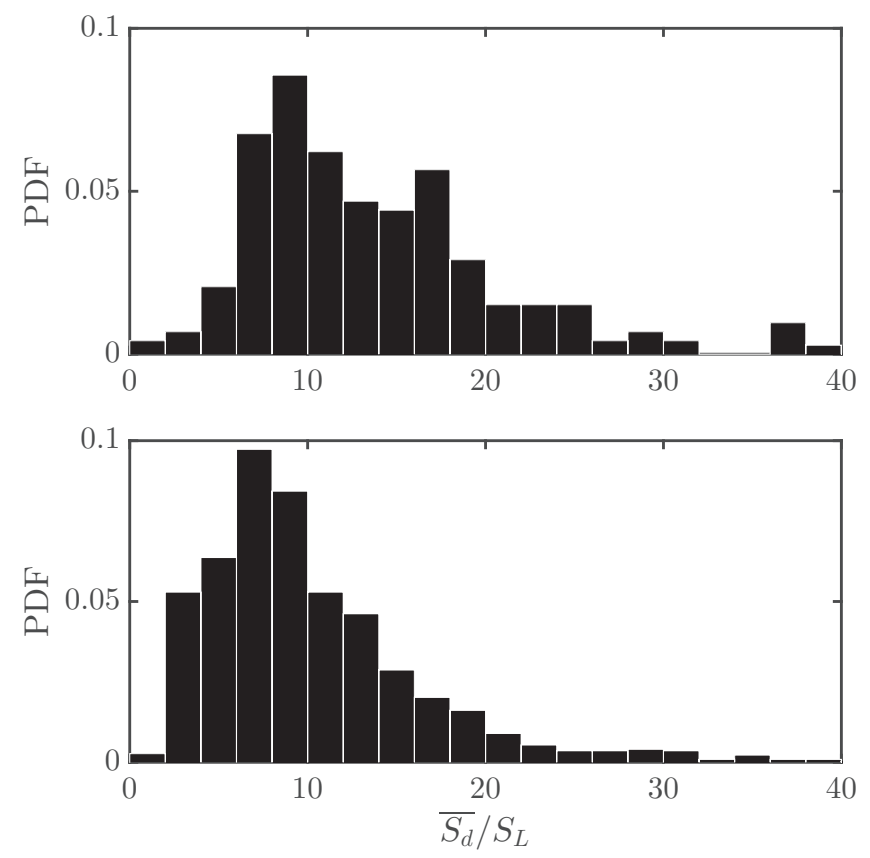

Figure 7: Histogram of the mean normalized flame displacement speed occurring in annihilation regions for $\phi=1$ (top) and $\phi=0.7$ (bottom).

tuations associated with tunnel formation (black squares) events feature a positive bias but the peak-to-peak amplitude is similar to the other two. Overall, different annihilation event types show similarity in terms of time scale and amplitude for a given equivalence ratio. This has implications for modelling flame interactions and suggests that the different topologies behave similarly in producing sound. This therefore supports the idea of the simple model proposed previously by Haghiri et al. [30], where all annihilation events were approximated as spherically symmetric.

A comparison between the $\phi=1.0$ and $\phi=0.7$ results shows a strong similarity, again implying a universality of flame annihilation behaviour. Note however that Figs. $8 \mathrm{a}$ and $8 \mathrm{~b}$ have different scales. The $\phi=0.7$ annihilation events have a smaller amplitude and occur over a longer period. This behaviour is likely the result of the lower $S_{L}$ (and therefore lower $S_{d}$ ) in lean combustion compared with that in stoichiometric combustion. This is supported by Eq. (13) and the scaling from Talei et al. [23], who found that the sound amplitude produced by a spherically symmetric annihilation event is proportional to $S_{L}^{2}$.

The ensemble averaged integral of $\partial \dot{Q} / \partial t$ in the annihilation regions for different canonical event types is shown in Fig. 9. The integrated $\partial \dot{Q} / \partial t$ again shows a weak dependence on to the event type, consistent with the observation in Fig. 8 and giving further evidence that a simplified approach to modelling the sound generation process is justified. 

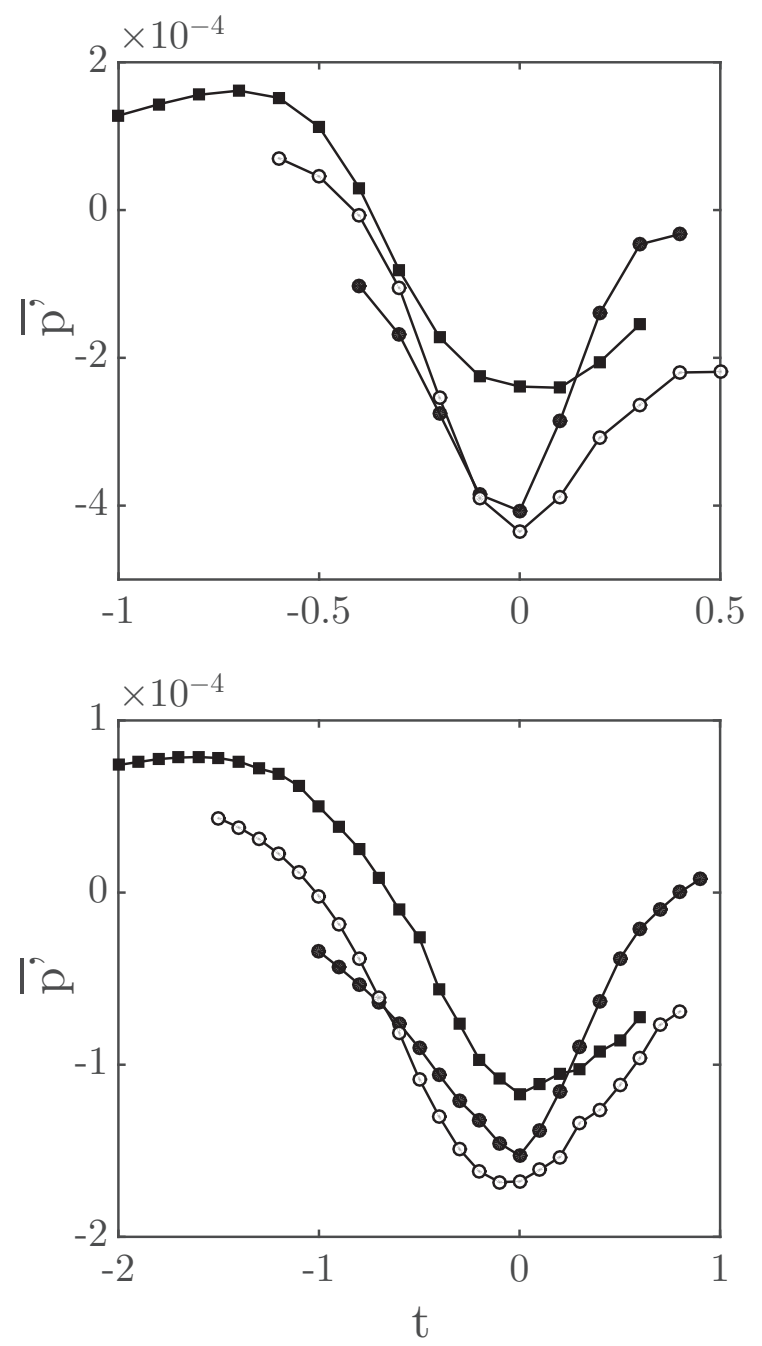

Figure 8: Temporal evolution of ensemble averaged pressure fluctuations at the critical points for pocket burn out events (black circles), tunnel closures (white circles) and tunnel formations (dark squares) for $\phi=1$ (top) and $\phi=0.7$ (bottom) $t=0$ corresponds to the instant when the flame iso-surfaces of maximum reaction rate collide.

\subsection{Sound generation by annihilation events}

Equation (5) is now used to compute the pressure fluctuations in the far field, by considering all computational cells. This approach will determine the generated sound by the fluctuations of heat release rate of the whole flame, i.e. the overall direct combustion noise. A second order interpolation in time was used to compute $\partial \dot{Q} / \partial t$ at time $t-\left|\mathbf{x}-\mathbf{x}_{i}\right| / c_{b}$.

The pressure fluctuations induced by $\partial \dot{Q} / \partial t$ and the equivalent DNS results are compared in Fig. 10. While both traces demonstrate strong correlation at location 1, the correlation weakens as the probe is moved closer to the inlet (location 3). This shows that other phenomena, such as inlet noise, play an important role in that region, as discussed in Haghiri et al. [30]. Nonetheless, 

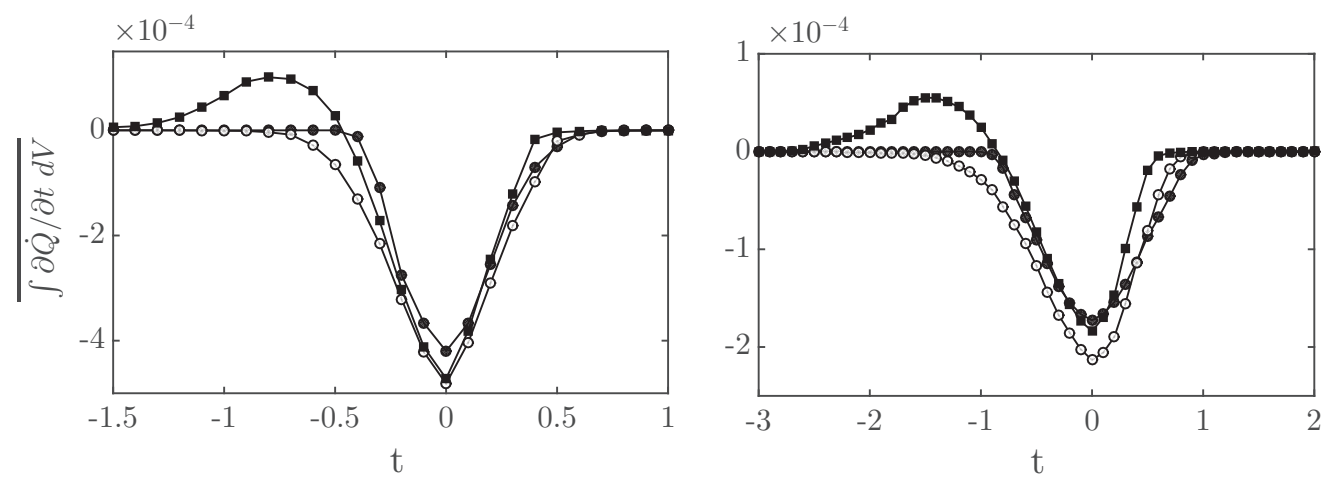

Figure 9: Temporal evolution of ensemble averaged $\int \partial \dot{Q} / \partial t d V$ over the observed annihilation regions for pocket burn out events (black circles), tunnel closures (white circles) and tunnel formations (dark squares) for $\phi=1$ (left) and $\phi=0.7$ (right).
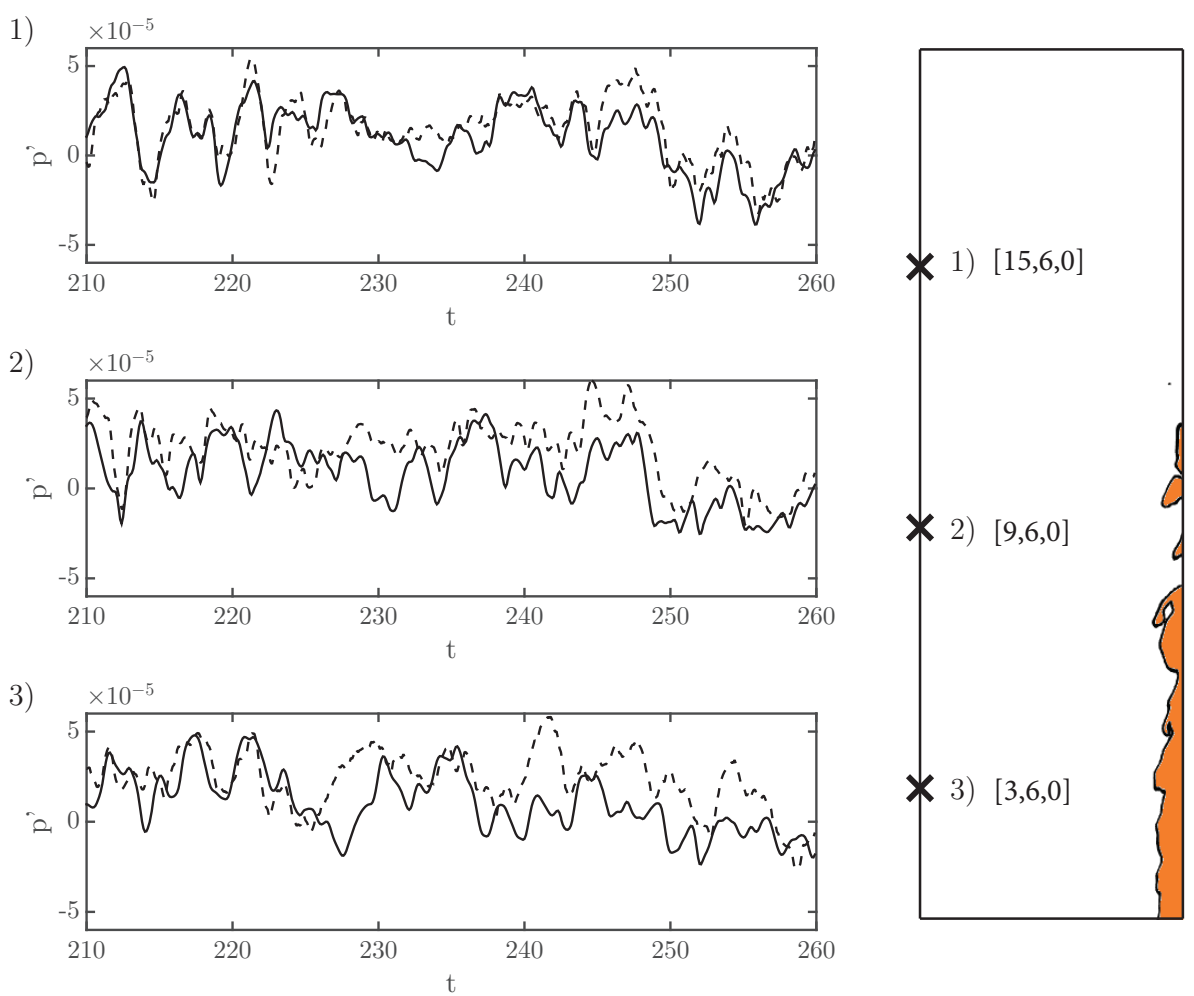

Figure 10: Pressure traces at the locations $[x, y, z]$ shown in the right panel, obtained from the DNS data (solid line) and Eq. (5) with the whole computational domain (dashed line), for $\phi=1$.

combustion noise is clearly dominant when the receiver is far enough from the inlet. The same analysis was performed on the $\phi=0.7$ flame and is shown in Fig. 11. The same qualitative 

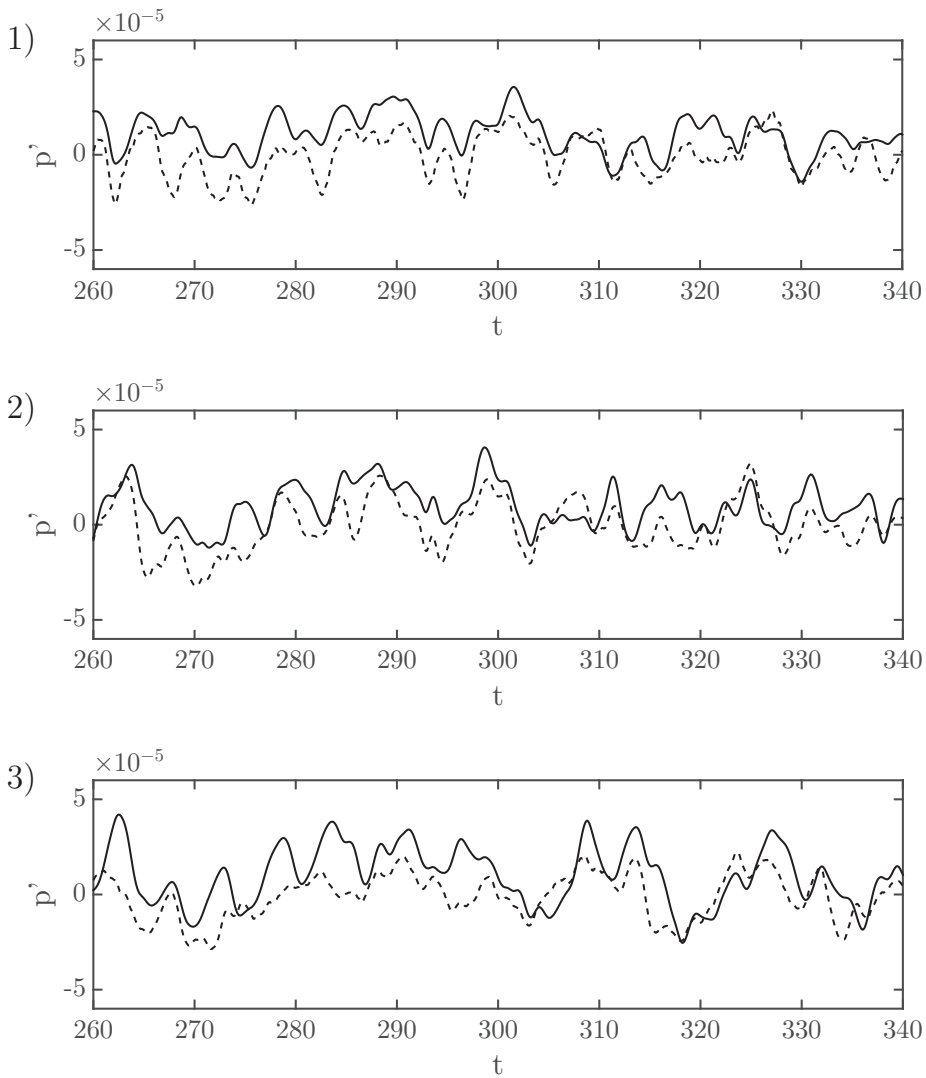

Figure 11: Pressure traces at locations shown in the right panel of Fig. 10, obtained from the DNS data (solid line) and Eq. (5) conditioned with the whole computational domain (dashed line), for $\phi=0.7$.

observations as those in Fig. 10 are made and therefore location 1 will be considered in the following analysis.

Figure 12 shows the spectra of the pressure fluctuations at location 1 from the DNS and those due to $\partial \dot{Q} / \partial t$ in the whole computational domain. The spectra were computed using 3 tapered cosine windows with a $50 \%$ overlap. The non-dimensional time interval $\Delta t$ was equal to 0.1 and each window had a length of 25 time units $(N=250$ samples $)$ and 40 time units $(N=400$ samples) for the stoichiometric and lean cases, respectively. The associated frequency range was therefore $\left[S t_{\text {min }} ; S t_{\text {max }}\right]=\left[f_{\text {min }} ; f_{\text {max }}\right]^{*} D / \bar{u}_{\text {in }}=[1 /(N \Delta t) ; 1 /(2 \Delta t)]^{*} D / \bar{u}_{\text {in }}$. Considering the Strouhal number being defined as $S t=f D / \bar{u}_{i n}$, where $f$ denotes the frequency, the range of Strouhal numbers were therefore equal to $[0.11 ; 14.3]$ and $[0.10 ; 19.2]$ for the stoichiometric and lean cases, respectively. The agreement of these two spectra to within $3 \mathrm{~dB}$ is confirmation that direct combustion noise via $\partial \dot{Q} / \partial t$ dominates the sound generation process.

Figure 13 shows the sound induced by the four different types of annihilation events at location 1 defined in Fig. 10, for both equivalence ratios. In this case, Eq. (5) is used to compute the far-field sound from the annihilation events while considering only the cells located inside the annihilation regions. Figure 13 also shows that tunnel closures and multi-feature events are the 

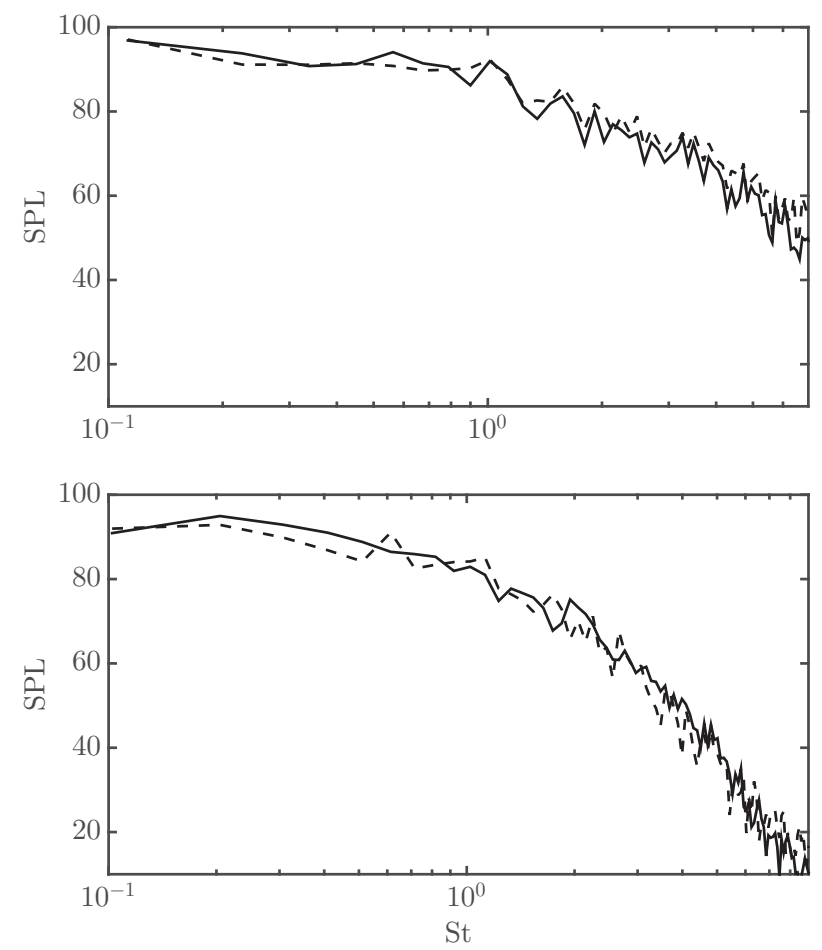

Figure 12: Pressure spectra at location 1 in Fig. 10, obtained from the DNS data (solid line) and Eq. (5) with the whole computational domain (dashed line), for $\phi=1$ (top) and $\phi=0.7$ (bottom).

dominant sources of noise whereas tunnel formation events are the weakest among all. This is not because of the relative strength of each event type as a source of sound, as discussed earlier in reference to Fig. 8 and 9, but because of the relative frequency of occurrence of each annihilation event. Thus, all annihilation event topologies are important to the sound generation process and event frequency rather than individual event strength appears important.

The overall contribution of these annihilation events to the far-field sound is now considered. To do this, Fig. 14 compares the spectra of the sum of the pressure traces presented in Fig. 13 to those calculated using Eq. (5) and the whole computational domain. Two features of Fig. 14 are of note. First, the stoichiometric and lean spectra have similar amplitude for $S t<1$, suggesting similar low frequency flame dynamics, but increasingly differ as $S t$ increases above unity. Furthermore, the close agreement between the two sets of spectra for a given $\phi$ and $S t>1$ is clear evidence that annihilation events are the dominant source of sound at these frequencies, even though there is some divergence at higher St and the smallest amplitudes for the lean case. The annihilation event timescale $\tau=\delta_{t h} / S_{d}$ is taken as $\tau=1.5$ and $\tau=3$ for the stoichiometric and lean cases respectively (see Fig. 8). This leads to a typical $S_{d}$ of $11 S_{L}$ and $7 S_{L}$ for the stoichiometric and lean cases, respectively. Figure 7 shows that displacement speeds of this magnitude are common during annihilation. In addition, the aforementioned annihilation timescale equates to Strouhal numbers $S t=D /\left(\tau \bar{u}_{i n}\right) \approx 1$. These points show that annihilation events are expected to have a strong acoustic signature around $S t=1$ and above. 
a)
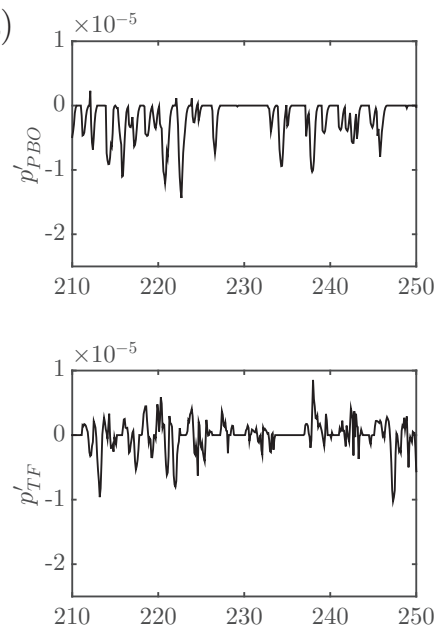

b)
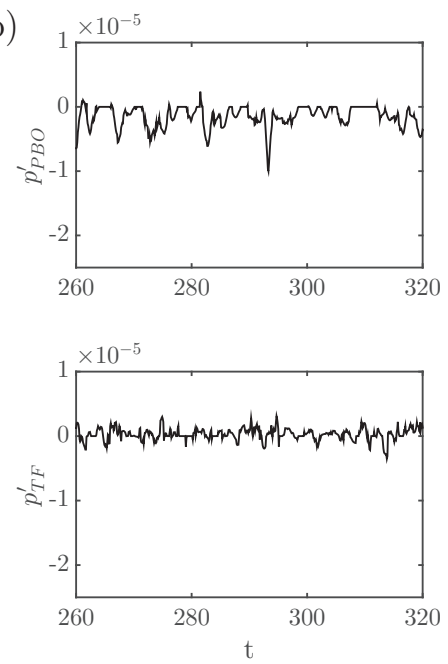
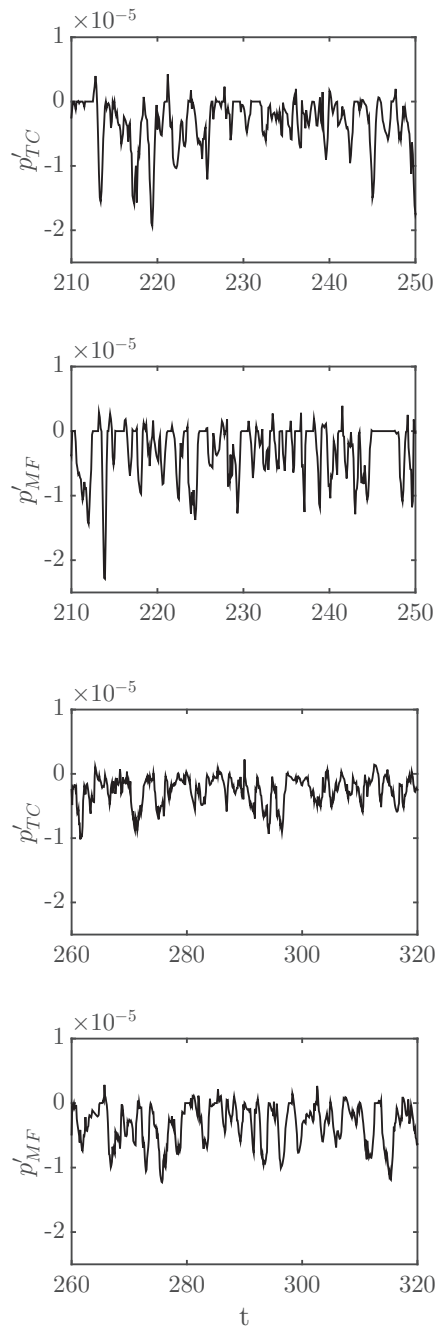

Figure 13: Pressure traces at location 1 in Fig. 10 for the pocket burn-out (top left), tunnel closure (top right), tunnel formation (bottom left) and multi-feature (bottom right) events, in the a) $\phi=1.0$ and b) $\phi=0.7$ flames.

The following scaling between the amplitude of the sound radiated by individual, canonical annihilation events to several fundamental flame parameters [23] suggests a dependence on $\phi$, particularly through variations in the temperature ratio $T_{b} / T_{u}$, the laminar flame speed $S_{L}$ and the thermal flame thickness $\delta_{t h}$,

$$
p^{\prime} \propto\left(S_{L}\right)^{1+(n / 2)}\left(\frac{\delta_{t h}}{\xi}\right)^{n / 2}\left(1-\frac{T_{u}}{T_{b}}\right)\left(\frac{T_{b}}{T_{u}}\right)^{(2-n) / 4},
$$

where $n=0$ for a planar case, $n=1$ for an axisymmetric case, $n=2$ for a spherically symmetric case and $\xi$ represents the distance between the annihilation event and the receiver. Thus, leaner 

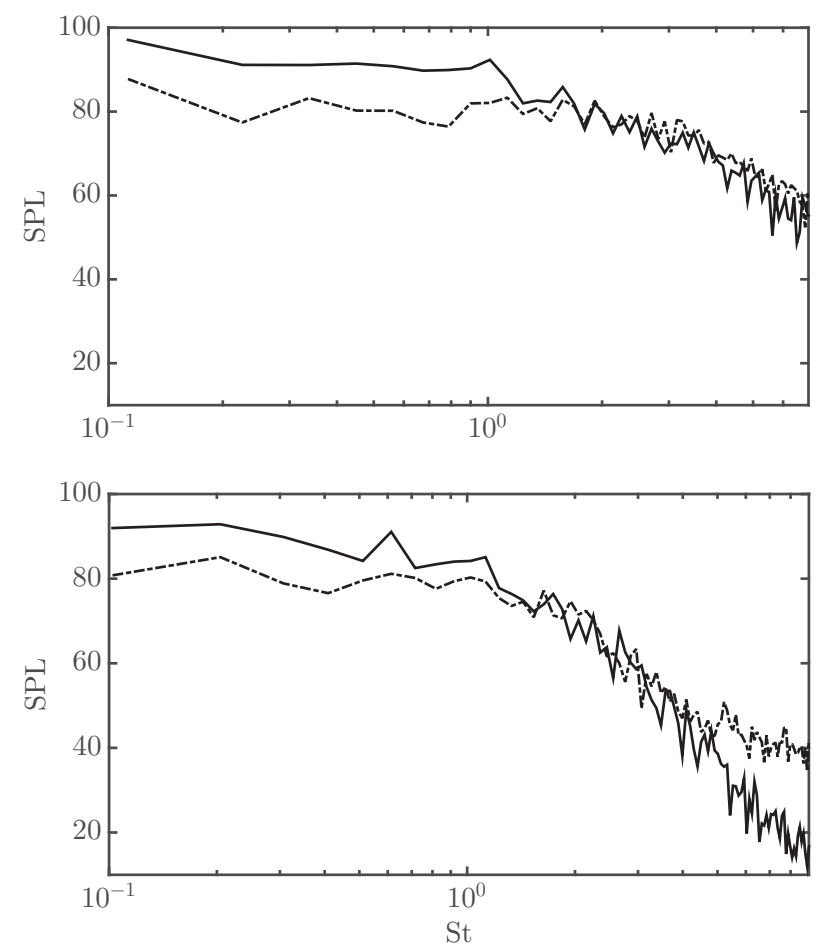

Figure 14: Pressure spectra at location 1 in Fig. 10 using Eq. (5) and considering either the whole computational domain (solid line) or the annihilation regions only (dot-dashed line) for $\phi=1$ (top) and $\phi=0.7$ (bottom).

combustion, characterized by lower $S_{L}$ and $T_{b}$, appears to result in a quieter flame via individual annihilation events becoming weaker acoustic sources.

\section{Conclusion}

This paper studied the contribution of flame annihilation events to the sound radiated by turbulent, premixed flames. Previously published direct numerical simulation (DNS) datasets of stoichiometric and lean $(\phi=0.7)$ flames [30] were first examined using an efficient formulation of the method of Griffiths et al. [31] to identify the annihilation events. Four classes of annihilation event were observed. Three of these - pocket burn-out, tunnel closure and tunnel formation were defined by Griffiths et al [31]. A 'multi-feature' event was also defined in this paper as any combination of the other three annihilation events occurring close enough such that their radiated sound can be considered as originating from a single event.

Further post-processing of these stoichiometric and lean datasets also showed that fluctuations in the heat release rate were the dominant source of flame noise, as several other studies have found. However, this study also showed that the fluctuations in heat release rate associated with the 4 observed types of annihilation events were responsible for the broadband sound radiated by both flames. This, in turn, suggested that flame annihilation is the physical mechanism by which air-fuel ratio affects the radiated sound amplitude at high frequencies. This result was supported 
by prior works by the group $[23,28]$ which have shown that the sound radiated from individual, canonical annihilation events scales with several fundamental flame parameters, in particular the laminar flame speed and the temperature ratio.

\section{Acknowledgements}

This work was supported by the Australian Research Council (ARC) [grants DP120101830 and DE180100416] and the University of Melbourne through a Melbourne International Research Scholarship and a Melbourne International Fee Remission Scholarship. The research benefited from computational resources provided through the National Computational Merit Allocation Scheme and the Pawsey Energy and Resources Scheme, supported by the Australian Government. The computational facilities supporting this project included the Australian NCI National Facility and the Pawsey Supercomputing Center.

\section{Appendix A. Interpolation method}

In order to apply Newton's optimization algorithm on a scalar field $c$, one must know the gradient vector $G$ and the Hessian matrix $\mathbf{H}$ at any point in space. When this method is applied to a cartesian grid, an interpolation is therefore required to interpolate between different cells. The convolution algorithm developed by Keys [41] can be used for this purpose. By using an interpolation kernel, which must be correctly defined, the discrete data can be converted into a continuous function. Meijering et al. [42] found the coefficients defining the best quintic convolution kernel. Using the matrix notation, the kernel can be used to define the interpolate value $q(x)$ for a 1-D case:

$$
q(x)=\frac{1}{2} X_{m} T_{m i} C_{i},
$$

where $X$ is the local coordinate vector $\left[\begin{array}{llllll}1 & x & x^{2} & x^{3} & x^{4} & x^{5}\end{array}\right]$, with $x$ between 0 and $1, C_{i}$ is the data vector $\left[\begin{array}{llllll}c_{-2} & c_{-1} & c_{0} & c_{1} & c_{2} & c_{3}\end{array}\right]$ containing values of $c$ at $x=\left[\begin{array}{llllll}-2 & -1 & 0 & 1 & 2 & 3\end{array}\right]$ , and $\mathbf{T}$ is the interpolation kernel matrix:

$$
\mathbf{T}=\frac{1}{64}\left[\begin{array}{cccccc}
0 & 0 & 64 & 0 & 0 & 0 \\
3 & -38 & 0 & 38 & -3 & 0 \\
-12 & 80 & -136 & 80 & -12 & 0 \\
18 & -36 & 0 & 36 & -18 & 0 \\
-12 & -19 & 126 & -144 & 46 & 3 \\
3 & 13 & -54 & 54 & -13 & -3
\end{array}\right]
$$

Note that Einstein summation applies in Eq. (A.1) and in the following. With this quintic formula, the interpolant will be element of $C^{3}$, ensuring continuity up to the third derivative. Equation (A.1) can be derived to find the first and second order derivatives of $q(x)$ :

$$
\begin{gathered}
\dot{q}(x)=\frac{1}{2} \dot{X}_{m} T_{m i} C_{i}, \\
\ddot{q}(x)=\frac{1}{2} \ddot{X}_{m} T_{m i} C_{i} .
\end{gathered}
$$


Equation (A.1) can be extended to a 3-D case by interpolating successively in the first, second and third dimensions, leading to the following formula:

$$
q(x, y, z)=\frac{1}{8} X_{m} Y_{n} Z_{p} T_{m i} T_{n j} T_{p k} C_{i j k} .
$$

The 3-D data tensor $C_{i j k}$ contains the information of the scalar field in a volume surrounding the interpolated cell. The gradient vector and the Hessian matrix components can then be computed by deriving the appropriate local coordinate vectors.

\section{References}

[1] J. W. S. B. Rayleigh, The theory of sound, Macmillan, 1896

[2] T. Poinsot, Prediction and control of combustion instabilities in real engines, Proc. Combust. Inst. 36 (2017) 1-28.

[3] T. Lieuwen, Unsteady combustor physics, Cambridge University Press, 2012.

[4] T. Schuller, Mécanismes de couplage dans les interactions acoustiques-combustion, Ph.D. thesis (2003).

[5] T. Schuller, D. Durox, S. Candel, Self-induced combustion oscillations of laminar premixed flames stabilized on annular burners, Combust. Flame 135 (2003) 525-537.

[6] A. P. Dowling, Y. Mahmoudi, Combustion noise, Proc. Combust. Inst. 35 (2015) 65-100.

[7] T. Poinsot, D. Veynante, Theoretical and Numerical Combustion, 3rd Edition, RT Edwards, Inc., 2005.

[8] S. Candel, Combustion dynamics and control: progress and challenges, Proc. Combust. Inst. 29 (2002) 1-28.

[9] M. Ihme, Combustion and engine-core noise, Annu. Rev. Fluid Mech. 49 (2017) 227-310.

[10] S. L. Bragg, Combustion noise, J. Inst. Fuel 36 (1963) 12-16.

[11] T. J. B. Smith, J. K. Kilham, Noise generation by open turbulent flames, J. Acoust. Soc. Am. 35 (1963) $715-724$.

[12] I. R. Hurle, R. B. Price, T. M. Sugden, A. Thomas, Sound emission from open turbulent premixed flames, Proc. R. Soc. London A Math. Phys. Eng. Sci. 303 (1968) 409-427.

[13] R. B. Price, I. R. Hurle, T. M. Sugden, Optical studies of the generation of noise in turbulent flames, Symp. (Int.) Combust. 12 (1969) 1093-1102.

[14] F. Briffa, C. J. Clark, G. T. Williams, Combustion noise, J. Inst. Fuel 46 (1973) 207-216.

[15] W. C. Strahle, B. N. Shivashankara, A rational correlation of combustion noise results from open turbulent premixed flames, Symp. (Int.) Combust. 15 (1975) 1379-1385.

[16] R. Rajaram, T. Lieuwen, Spatial distribution of combustion noise sources, 13th AIAA/CEAS Aeroacoustics Conf. (2007) 3698.

[17] R. Rajaram, T. Lieuwen, Acoustic radiation from turbulent premixed flames, J. Fluid Mech. 637 (2009) $357-385$.

[18] C. K. W. Tam, The spectral shape of combustion noise, Int. J. Aeroacoustics 14 (2015) 431-456.

[19] H. Chiu, M. Summerfield, Theory of combustion noise, Acta Astronaut. 1 (1974) 967-984.

[20] W. C. Strahle, A more modern theory on combustion noise, in: Recent Advances in the Aerospace Sciences, Springer, 1985, pp. 103-114.

[21] A. P. Dowling, Thermoacoustic sources and instabilities, in: Modern Methods in Analytical Acoustics, Vol. 92 , Springer-Verlag, 1992, pp. 378-405.

[22] A. Thomas, G. T. Williams, Flame noise: sound emission from spark-ignited bubbles of combustible gas, Proc. R. Soc. London A Math. Phys. Eng. Sci. 294 (1966) 449-466.

[23] M. Talei, M. J. Brear, E. R. Hawkes, Sound generation by laminar premixed flame annihilation, J. Fluid Mech. 679 (2011) 194-218.

[24] N. Kidin, V. Librovich, J. Roberts, M. Vuillermoz, On sound sources in turbulent combustion, Dyn. Flames React. Syst. 95 (1984) 343-355.

[25] N. Kidin, V. Librovich, M. Macquisten, J. Roberts, M. Vuillermoz, Possible acoustic source in turbulent combustion, Dyn. React. Syst. Part 1 (1988) 336-348.

[26] S. Candel, D. Durox, T. Schuller, Flame interactions as a source of noise and combustion instabilities, 10th AIAA/CEAS Aeroacoustics Conf. (2004) 2928.

[27] M. Talei, M. J. Brear, E. R. Hawkes, A parametric study of sound generation by premixed laminar flame annihilation, Combust. Flame 159 (2012) 757-769.

[28] M. Talei, E. R. Hawkes, M. J. Brear, A direct numerical simulation study of frequency and Lewis number effects on sound generation by two-dimensional forced laminar premixed flames, Proc. Combust. Inst. 34 (2013) 1093-1100.

[29] M. Talei, M. J. Brear, E. R. Hawkes, A comparative study of sound generation by laminar, combusting and noncombusting jet flows, Theor. Comput. Fluid Dyn. 28 (2014) 385-408. 
[30] A. Haghiri, M. Talei, M. J. Brear, E. R. Hawkes, Sound generation by turbulent premixed flames, J. Fluid Mech. 843 (2018) 29-52.

[31] R. A. C. Griffiths, J. H. Chen, H. Kolla, R. S. Cant, W. Kollmann, Three-dimensional topology of turbulent premixed flame interaction, Proc. Combust. Inst. 35 (2015) 1341-1348.

[32] J. H. Chen, A. Choudhary, B. De Supinski, R. DeVries, E. R. Hawkes, S. Klasky, W. K. Liao, K. L. Ma, J. MellorCrummey, N. Podhorszki, R. Sankaran, S. Shende, C. S. Yoo, Terascale direct numerical simulations of turbulent combustion using S3D, Comput. Sci. Discov. 2 (2009) 015001.

[33] S. Karami, E. R. Hawkes, M. Talei, J. H. Chen, Edge flame dynamics in a turbulent lifted jet flame, Proceedings of the 2014 Summer Program, Center for Turbulence Research (Stanford, CA) (2015) 137-146.

[34] S. Karami, E. R. Hawkes, M. Talei, J. H. Chen, Mechanisms of flame stabilisation at low lifted height in a turbulent lifted slot-jet flame, J. Fluid Mech. 777 (2015) 633-689.

[35] S. Karami, E. R. Hawkes, M. Talei, J. H. Chen, Edge flame structure in a turbulent lifted flame : A direct numerical simulation study, Combust. Flame 169 (2016) 110-128.

[36] T. Poinsot, S. K. Lele, Boundary conditions for direct simulations of compressible viscous flows, J. Comput. Phys. 101 (1992) 104-129.

[37] C. S. Yoo, H. G. Im, Characteristic boundary conditions for simulations of compressible reacting flows with multidimensional, viscous and reaction effects, Combust. Theory Model. 11 (2007) 259-286.

[38] T. Colonius, S. K. Lele, P. Moin, Sound generation in a mixing layer, J. Fluid Mech. 330 (1997) 375-409.

[39] M. J. Lighthill, On sound generated aerodynamically. I. General theory, Proc. R. Soc. London A Math. Phys. Eng. Sci. 211 (1952) 564-587.

[40] T. Echekki, J. H. Chen, Analysis of the contribution of curvature to premixed flame propagation, Combust. Flame 311 (1999) 308-311.

[41] R. Keys, Cubic convolution interpolation for digital image processing, IEEE Trans. Acoust. 29 (1981) 1153-1160

[42] E. H. W. Meijering, K. J. Zuiderveld, M. A. Viergever, Image reconstruction by convolution with symmetrical piecewise nth-order polynomial kernels, IEEE Trans. Image Process. 8 (1999) 192-201. 